\title{
肠道隐窝-线毛轴上皮细胞更新及调控机制研究 进展
}

\author{
邹立军 ${ }^{1,2,3}$, 熊霞 ${ }^{2 *}$, 王小城 ${ }^{2}$, 杨焕胜 ${ }^{1}$, 李建中 ${ }^{1}$, 黄鹏飞 ${ }^{1}$, 印遇龙 ${ }^{1,2^{*}}$ \\ 1. 湖南师范大学生命科学学院, 动物营养与人体健康实验室, 长沙 410081 ; \\ 2. 中国科学院亚热带农业生态研究所, 亚热带农业生态过程重点实验室, 长沙 410125; \\ 3. 湖南第一师范学院, 基础生物学实验室, 长沙 410205 \\ *联系人, E-mail: xx@isa.ac.cn; yinyulong@isa.ac.cn
}

收稿日期: 2016-11-15; 接受日期: 2016-11-27; 网络版发表日期: 2017-01-04

国家自然科学基金(批准号: 31572420,31301988,31272261)和湖南省研究生科研创新项目(批准号: CX2016B172)资助

\begin{abstract}
摘要肠道不仅是营养物质消化吸收的主要部位, 也是重要的免疫器官和内分泌器官. 小肠上皮细胞的分化 对于肠道应激后的损伤修复、免疫屏障以及肠道功能的正常行使具有非常重要的意义. 近年来, 肠道上皮隐窝 一线毛轴千细胞自我更新、分化和调控的研究得到了快速发展. 本文结合本研究组的研究成果综述了哺乳动 物肠道隐窝-线毛轴上皮细胞分化过程中差异基因和蛋白表达; 信号通路、转录因子和表观遗传修饰对肠上皮 细胞分化的影响以及营养因子对肠道细胞分化和损伤修复调控的最新研究进展, 以期在营养学和药理学方面, 为千预和治疗肠道损伤及相关疾病提供理论指导依据.
\end{abstract}

关键词小肠, 隐窝-线毛轴, 细胞更新, 损伤修复, 营养调控

肠道不仅是营养物质消化吸收的主要部位, 也是 重要的免疫器官和内分泌器官 ${ }^{[1]}$. 肠道稳态维持是通 过肠道干细胞(intestinal stem cell)的增殖分化实现的, 肠上皮细胞沿隐窝端向线毛端迁移, 逐渐分化成熟并 最终在线毛顶端脱落, 分化成熟后的肠上皮细胞有着 显著的顶角结构和上千个微线毛 ${ }^{[2]}$. 小肠上皮作为哺 乳动物更新速度最快(4 5天)的器官, 其分化对于肠道 应激后的损伤修复、肠道屏障以及肠道功能的正常行 使具有非常重要的意义 ${ }^{[3]}$. 小肠隐窝干细胞具有非常 精细的细胞增殖与分化的调控机制, Wnt/ $\beta$-catenin、
Notch、表皮生长因子(epidermal growth factor, EGF)和 骨形成蛋白(bone morphogenetic protein, BMP)等信号 在小肠干细胞命运调控中起着非常重要的作用 ${ }^{[4]}$. 结 合本研究组的成果, 本文综述了哺乳动物肠道隐窝-线 毛轴(crypt-villus axis)细胞的组成; 肠上皮细胞增殖分 化和损伤修复过程中相关基因、蛋白的表达差异; 信 号通路、转录因子和表观遗传修饰对肠上皮细胞分 化的影响; 并从营养生理学的角度总结了各类营养调 控因子促进肠道细胞增殖分化和肠道损伤修复等方 面的研究进展.

引用格式: 邹立军, 熊霞, 王小城, 等. 肠道隐窝-线毛轴上皮细胞更新及调控机制研究进展. 中国科学: 生命科学, 2017, 47: 190-200 Zou L J, Xiong X, Wang X C, et al. Current advances in renewal mechanisms of intestinal epithelial cells along the crypt-villus axis. Sci Sin Vitae, 2017, 47: 190-200, doi: 10.1360/N052016-00240 


\section{1 小肠隐窝-线毛轴上皮细胞组成}

肠道上皮由单层柱状上皮细胞层覆盖并可分为 隐窝和线毛两部分; 隐窝内陷进入了下层间充质, 线 毛朝向肠腔 ${ }^{[5]}$. 肠上皮细胞快速和持续的更新动力依 赖于隐窝处的Lgr5阳性(Lgr5 $5^{+}$)特异标记的底部柱状细 胞( $\mathrm{CBC}$ 细胞 (crypt base columnar cell)), $\mathrm{CBC}$ 细胞(干细 胞)分裂增殖后产生的子代细胞继续分化为“短暂扩增 细胞”(TA细胞(transit amplifying cell))并继续向上迁移 分化 ${ }^{[6]}$. 因此, 隐窝和线毛分别包括干细胞增殖和分化 细胞区域. 分化成熟的肠上皮细胞主要包括4种类型的 细胞: 吸收型上皮细胞(absorptive enterocytes)和3种分 泌型细胞(secretory lineages). 吸收型肠上皮细胞主要 负责营养物质的吸收, 其数量约占线毛细胞的 $95 \%$ 以 上 $^{[7]}$. 分泌型肠上皮细胞与肠道免疫屏障有密切关系, 其中, 杯状细胞(goblet cells)在整个上皮分布并分泌黏 液; 潘氏细胞 (paneth cells) 在隐窝底部聚集, 产生抗菌 肽并调节肠道微生物, 同时也产生生长因子维持附近 干细胞的生长; 肠内分泌细胞 (enteroendocrine cells)数 量较少, 但负责调节肠道上皮的多种功能, 如分泌激 素和消化酶调控饱腹感等功能 ${ }^{[8,9]}$. 此外, 肠道上皮还 存在 $M$ 细胞 (membranous cell)和塔夫细胞(tuft cells). M 细胞存在于肠道相关的淋巴滤泡, 可能与肠腔内物质 与免疫细胞的交流有关 ${ }^{[10]}$. 塔夫细胞在肠道上皮中的 数量较少, 目前它们的功能尚不清楚 ${ }^{[11]}$.

分化形成的各种细胞(除潘氏细胞外)在沿隐窝线毛轴向上移动的过程中逐渐成熟, 最后达到线毛顶 端区域并最终调亡 ${ }^{[2]}$. Canale-Zambiano等人 ${ }^{[12]}$ 发现, DNA合成在隐窝-线毛过渡期间迅速下降, 表明分化 过程中伴随着细胞周期阻滞. 肠道某些特定基因的 转录以及线毛顶端细胞存在DNA碎片, 暗示着有细 胞调亡的发生, 这表明肠上皮细胞的衰老和调亡是一 个有序过程 ${ }^{[13]}$. 但关于线毛顶端mRNA表达的下降和 细胞衰老之间的关系还需要进一步研究. 另外, 小肠 线毛细胞的脂质过氧化程度显著高于隐窝, 在线毛区 域产生大量自由基, 这可能是导致肠上皮细胞从线毛 顶端脱落发生调亡的原因之一, 这也促进了肠上皮细 胞的分化迁移 ${ }^{[14]}$. 研究表明, 细胞平面极性信号通路 (the planar cell polarity, PCP) 对上皮细胞极性的建立和 细胞迁移有重要作用; 其作用机制主要是通过激活 小 $\mathrm{G}$ 蛋白 RhoA和Rac1后再分别诱导下游Rho-相关激
酶(Rho-associated coiled-coil protein kinase, ROCK) 和 c-Jun氨基末端激酶(c-Jun N-terminal kinas, JNK)的表 达进而分别诱导细胞骨架重塑或引起转录反应 ${ }^{[15]}$. 因 此, 小肠上皮细胞沿线毛分化迁移和在线毛上脱落是 个复杂严密的过程.

\section{2 肠道隐窝-线毛轴细胞基因及蛋白表 达变化}

\section{1 消化吸收相关基因和蛋白的表达差异}

肠道刷状缘是营养物质消化吸收的最终部位, 肠 上皮细胞分化程度越高, 它的消化吸收能力越强. 肠 碱性磷酸酶(alkaline phosphatase, ALP) 是营养物质吸 收的标志酶, 与蛋白质和脂质的代谢有密切关系, 其 表达量和活性在肠上皮细胞顶端处最高, 隐窝区域最 低 ${ }^{[16]}$. 三磷酸腺苷酶 $\left(\mathrm{Na}^{+} / \mathrm{K}^{+}\right.$-ATPase $)$是细胞膜上的糖 蛋白, 主要作用是通过参与维持肠道上皮细胞膜内外 的离子浓度差来吸收营养物质, 其表达沿隐窝-线毛 轴从上到下逐渐降低 ${ }^{[17]}$. 一系列研究表明, 二糖酶蔗 糖酶-异麦芽糖酶(sucrase isomaltase, SI)、乳糖酶-根 皮苷水解酶(lactase-phlorizin hydrolase, LPH)、氨肽酶 $\mathrm{N}$ (aminopeptidasen, APN)、蔗糖酶(invertase)和乳糖分 解酶(lactase, Lct)等酶类在线毛上的表达和活性均高 于隐窝 $[7,18]$.

负责营养物质吸收转运的转运载体在隐窝-线 毛轴的分布也存在差异, 如溶质载体家族基因在隐 窝-线毛轴上的表达不同 ${ }^{[19]}$. 易化性单糖转运体 5(facilitated glucose transporter 5, GLUT-5)/溶质载体家族2 成员 5(solute carrier family 2 member 5, SLC2a5) 和 $\mathrm{Na}^{+}$ 依赖性单糖转运体 $1\left(\mathrm{Na}^{+}\right.$-dependent glucose transporter, SGLT1) 是哺乳动物体内的两种单糖转运体, 对碳水 化合物的吸收起着重要作用. 研究表明, 大鼠(Rattus norvegicus) GLUT-5 在线毛3/4以上部位表达 ${ }^{[20]}$. SGLT1 在线毛部位的表达量高于隐窝区域,而其蛋白质的表 达却没有差异, 表明隐窝-线毛轴上的基因表达可能存 在转录后或翻译后水平的调控 ${ }^{[21]}$.

通过DNA微矩阵检测大鼠空肠隐窝-线毛轴细胞 分化的实验表明, 从隐窝干细胞分化形成线毛细胞的 过程中与消化吸收相关的基因表达显著上调, 如单羧酸 转运蛋白(SLC16a3)、钠偶联核苷转运蛋白(SLC28a1) 
和铜转运蛋白 (SLC31a1) 等; 与淀粉和蔗糖的消化吸 收有关的基因, 如 $S L C 2 a 5, S L C 5 a 1$ 和 $S I$ 等在线毛中端 表达最高; 而与脂肪吸收相关的基因, 如脂肪酸结 合蛋白(fatty acid-binding protein 1, Fabpl)、载脂蛋白 C3(apolipoprotein C3, Apoc3)和载脂蛋白A4(apolipoprotein A4, Apoa4)等的表达在线毛顶部表达最高 ${ }^{[22]}$. 肠 脂肪酸结合蛋白(intestines fatty acids binding proteins, I-FABP) 对肠道细胞内脂肪酸的利用过程起重要作 用, 蛋白质组学和免疫荧光实验均证明 I-FABP在整 个隐窝-线毛轴上均有表达, 且在线毛顶端的表达量 最高 ${ }^{[3,23]}$. 因此, 营养物质和离子的消化吸收基因在隐 窝-线毛轴上表达模式的差异表明肠道不同部位的肠 黏膜可能对这些过程有不同的贡献. 在哺乳动物中, 肠道沿隐窝-线毛轴上基因表达模式的变化与上皮细 胞更新之间的关系需要进一步研究, 以提高对营养物 质和离子消化吸收的认识.

\section{2 肠道细胞损伤修复过程中的基因和蛋白质表 达变化}

哺乳动物 (大鼠、猪(Sus scrofa domestica) 等)的 早期断奶和细胞感染均能引起肠道功能障碍和损 伤. 首先, 严重影响肠道的形态结构, 引起小肠黏 膜的损伤, 包括线毛萎缩以及隐窝增生, 导致ALP 的表达和活性下降, 增加肠道疾病的易感性 ${ }^{[24]}$. 其 次, 能够影响肠道功能基因的表达, 全基因组芯片 分析早期断奶仔猪肠道差异表达基因, 发现与调亡 相关基因, 如细胞色素 C(cytochrome C, CYCS), Bcl$\mathrm{xl} / \mathrm{Bcl}-2$ 相关死亡启动子 $(\mathrm{bcl}-\mathrm{xl} / \mathrm{bcl}-2$ associated death promoter, $B A D)$ 和共济失调毛细血管扩张症突变基因 (ataxia telangiectasia-mutated gene, $A T M$ )、促炎因子白 细胞介素-8(interleukin- $8, I L-8$ ) 和肿瘤坏死因子(tumor necrosis factor, $T N F)$ 表达上调, 而细胞周期调控相关 基因 $\mathrm{E} 2 \mathrm{~F}$ 转录因子 $5(\mathrm{E} 2 \mathrm{~F}$ transcription factor 5,E2F5) 和 SMAD家族成员 4(SMAD family member 4,Smad4)表达 下调; 表明断奶引起免疫抑制, 使细胞周期停滞, 加速 调亡 ${ }^{[25]}$. 在脂多糖(lipopolysaccharides, LPS)诱导的仔 猪肠道疾病模型实验中发现, 肠道免疫反应、黏膜生 长、能量代谢、吸收、黏膜屏障功能、抗病毒功能 等相关基因的表达均受到影响 ${ }^{[26]}$. 另外, 断奶还使仔 猪抗氧化能力显著下降; 肠道细胞内与氧化应激相关 基因表达增加, 自由基代谢异常, 血清中 $\mathrm{NO}$ 和 $\mathrm{H}_{2} \mathrm{O}_{2}$ 含
量显著增加, 而体内自由基水平与肠道结构与功能有 密切关联 ${ }^{[27]}$

本研究组以哺乳动物猪为实验材料, 建立了仔猪 肠道沿隐窝-线毛轴细胞分级方法, 利用碱性磷酸酶 和增殖细胞核抗原验证进行了哺乳阶段仔猪空肠沿 隐窝-线毛轴的蛋白质组学相关研究, 结果显示, 在肠 道细胞分化过程中, 结构和酶调节的蛋白质表达显著 下调; 而对营养物质的消化和吸收相关蛋白质的表达 上调 ${ }^{[3]}$, 这是由于在猪肠上皮细胞快速更新的过程中 对能量有显著需求所致 ${ }^{[7]}$; 而饲粮中的氨基酸(如谷氨 酸、天冬氨酸和谷氨酰胺)、葡萄糖和脂肪酸是猪肠 黏膜的主要能量来源. 通过断奶和哺乳仔猪相比, 发 现抗氧化能力随着肠上皮细胞分化而逐渐降低; 隐窝 -线毛轴的差异蛋白主要表现在参与碳代谢、氨基酸 和蛋白质代谢、磷代谢和氧化磷酸化等(数据未发表); 参与 $\beta$-氧化和糖酵解相关蛋白在线毛细胞内的表达下 降, 而糖酵解相关蛋白在隐窝区域表达上调, 表明能 量代谢在小肠细胞的增殖和分化过程中发挥了重要 作用 ${ }^{[16]}$. 因此, 对肠道损伤机制及其营养调控修复的 研究日益受到关注。

\section{3 小肠隐窝-线毛轴细胞分化的分子调控}

\section{1 调控小肠干细胞增殖分化的信号通路}

小肠上皮细胞更新是由多种信号通路进行严格 调控, 例如, Wnt, Notch, BMP和EGF等信号通路在维 持小肠干细胞的自我更新、增殖和分化方面有重要 作用. 上述信号通路调控小肠干细胞沿隐窝-线毛轴 分化的调控机制在Qi和 $\mathrm{Chen}^{[4]}$ 的报道中已有详细阐 述, 在此主要从Wnt和Notch信号通路协同调控的角度 进行综述. 研究表明, Wnt信号作为维持小肠上皮细胞 持续更新的主要动力, 其活性在小肠线毛-隐窝轴上 呈梯度递减性 ${ }^{[28]}$; 而Notch信号的活性是决定干细胞 分化为吸收型细胞和分泌型细胞的关键因素 ${ }^{[29]}$. 最新 研究证明,Wnt和Notch信号通路能协同调控肠道干细 胞的自我更新和分化. Yin等人 ${ }^{[30]}$ 通过篮选小鼠 $(M u s$ musculus) $\mathrm{Lgr}^{+}$干细胞进行体外 3D培养, 并利用小分 子调控Wnt和Notch信号通路, 从而控制肠道干细胞自 我更新和分化的方向; 结果表明, Wnt和Notch信号一 起激活能够维持干细胞自我增殖; 抑制Wnt信号, 激 
活Notch信号可定向诱导肠上皮细胞分化; 激活Wnt信 号, 抑制Notch信号可定向诱导潘氏细胞分化; 同时抑 制Wnt和Notch信号则定向诱导杯状细胞分化; 而内分 泌细胞分化需要抑制Notch信号通路, 但Wnt通路对其 影响不大 ${ }^{[31]}$. 因此, 信号通路的协同调控对维持小肠 干细胞的正常功能以及肠上皮细胞之间的分化平衡 起重要作用.

\section{2 细胞分化相关转录因子调控}

为了进一步了解隐窝-线毛轴细胞更新过程中的 基因差异表达的机制, 就必须探索基因与信号转导和 转录因子之间的联系. Mariadason等人 ${ }^{[32]}$ 通过DNA芯 片技术检测小鼠肠道隐窝-线毛轴肠上皮细胞差异基 因的表达, 结果显示, 随着细胞的分化迁移, 与细胞周 期进程、RNA转录和翻译相关的基因在成熟过程下 调, 与细胞骨架组装和脂质摄入相关的基因上调, 其 中Wnt信号基因表达差异最大, 其靶基因C-myc癌基 因 (myc proto-oncogene protein, $M Y C$ ) 和细胞周期蛋白 D1 ( cyclin D1)等在线毛顶端表达量最低. 随后发现, 在 小鼠肠道细胞中转录因子胰腺和十二指肠同源基因 (pancreas/duodenum homeobox protein 1, $P d x l$ ) 和甲状 腺激素受体 (thyroid hormone receptor $\alpha, T h r \alpha$ ) 等沿隐窝 -线毛轴表达下调, 在线毛顶端处表达量最低 ${ }^{[33]}$, 而蛋 白磷酸酶(protein phosphatase 5C, Ppp 5c) 隐窝处表达最 高 ${ }^{[21]}$, 而 $P p p 5 c$ 作为一种重要的核转录因子能通过诱 导 $P d x l$ 和 $T h r a$ 的表达 ${ }^{[34]}$, 随后负调控靶基因 $S I$ 和 $L c t$ 在 隐窝-线毛轴上表达显著升高 ${ }^{[35]}$. 结果表明, 肠道转录 因子相关基因的差异表达在小肠上皮细胞分化过程 中能够调控与细胞生长和消化吸收相关基因的表达.

尾型同源框转录因子2(homeobox protein CDX-2, $c d x 2$ ) 作为肠上皮细胞特异性核转录因子, 大量表 达在线毛末端处, 主要调控肠道上皮的增殖分化和 迁移 ${ }^{[36]}$; Shimakura等人 ${ }^{[37]}$ 发现, 人肠道上皮细胞中 氢/肽偶联转运载体Pept1(peptide transporter PEPT1, $S L C 15 A 1$ ) 基因表达受 $c d x 2$ 调控, 由于Pept1启动子上 没有 $c d x 2$ 经典的 TA序列结合区域从而通过结合转录 因子 $s p 1$ 上的启动子区域间接地调控Pept 1 表达. 小鼠 上皮细胞中 $c d x 2$ 可结合到短链脂肪酸转运载体(solute carrier family $5 \mathrm{~A}$ member $8, S L C 5 A 8$ ) 启动子区域, 上调 其mRNA表达 ${ }^{[38]} . c d x 2$ 除了参与调控上述与肠道营养 物质转运相关基因表达外, 还参与一些其他关于肠
道上皮细胞增殖分化和肠道上皮结构基因的表达调 控. Yamamoto等人 ${ }^{[39]}$ 证明, $c d x 2$ 能够通过结合肠道黏 蛋白基因(Mucin-2, MUC2)的顺式作用原件来激活其 表达, 而 $M U C 2$ 在杯状细胞中特异表达, 因此 $c d x 2$ 在杯 状细胞分化中起重要作用. 作为钙依赖型凝集素超家 族的一员再生基因 4(regenerating islet-derived protein 4, RegIV), 是表皮生长因子受体 (epidermal growth factor receptor, EGFR)/Akt/actibator protein-1信号通路潜在的 激活者, 在结直肠肿瘤中异位高表达; $c d x 2$ 可以直接结 合到Reg $I V$ 的启动子区域并且调控其表达 ${ }^{[40]}$. Coskun 等人 ${ }^{[41]}$ 分析了与 $c d x$ 结合相关的全基因组启动子, 发 现细胞吞噬运动蛋白 3 (engulfment and cell motility protein 3,ELMO3) 是 $c d x 2$ 的潜在靶基因, 并验证了 $c d x 2$ 和 $s p 1$ 能够协同调控 $E L M O 3$ 基因的表达, 从而调控细胞 的迁移. 最新研究表明, $c d x 2$ 能够直接结合到氨基酸 转运蛋白基因 $S L C 7 A 7$ 启动子区域并激活其表达从而 诱导猪小肠上皮细胞增殖 ${ }^{[42]}$.

转录因子GATA家族属于锌指蛋白家族, GATA-4/5/-6在成熟的小肠上皮细胞内表达, GATA-4在小肠的 近端到远端呈由高至低的表达梯度, 而 GATA-5和 6 在 空肠和回肠高表达, 它们能抑制或激活肠道特异性基 因 Lct, Invertase, I-FABP 和顶端钠离子/胆汁酸转运体 (apical sodium-dependent bile acid transporter, $A S B T$ ) 的 表达 ${ }^{[43-45]}$. GATA-4和 GATA-6在肠上皮细胞结构和细 胞分化中起着重要的作用; 成年小鼠小肠上皮中条件 性敲除 GATA-4和 GATA- 6 引起十二指肠和空肠内干细 胞增殖, 肠内分泌细胞和潘氏细胞显著减少, 而杯状 细胞大量增加 ${ }^{[46]}$. Walker等人 ${ }^{[47]}$ 证明, GATA转录因子 通过对Notch信号配体Dll1 的精细调控来抑制分泌型 细胞的分化. 另外, $c d x 2$ 还与 $G A T A-4$ 和肝细胞核因子 $-1 \alpha$ (hepatocyte nuclear factor- $1 \alpha, H N F-1 \alpha$ )协同作用调控 肠道细胞分化的开始并参与肠道上皮结构发育的调 整 ${ }^{[48,49]}$.

\section{3 表观遗传修饰对肠道细胞分化的调控}

表观遗传修饰不仅可以影响细胞基因的表达,而 且这种影响还可随细胞分裂而遗传. 近年来, 表观遗传 调控干细胞的分化机制已成为研究热点和前沿领域. 最近的研究表明,基因表达的骤变经常发生在细胞分化 过程中,并且是伴随着主要的染色质结构变化所引发的 修饰, 如组蛋白尾部的乙酰化和基因甲基化. 研究发现, 
组蛋白去乙酰化酶1(histone deacetylase 1, HDAC1)、组 蛋白乙酰转移酶2(histone acetyltransferase 2, Myst2)、 CREB 结合蛋白(CREB binding protein, $C B P$ ) 和E1A结 合蛋白p300(E1A binding protein p300,EP300)在沿小 鼠肠道隐窝-线毛轴的表达量迅速下降; 这些基因在肠 道细胞分化过程中与 $S I$ 和SGLTI的启动子/增强子区域 结合从而诱导其高表达. DNA甲基化主要发生在CpG 双核苷酸序列的胞嘧啶上; 肠道细胞更新的研究主要 集中在DNA甲基化的变化伴随着隐窝干细胞分化 ${ }^{[50]}$ 以及DNA 甲基化在早期是如何介入并控制细胞的分 化 ${ }^{[51]}$. Ziller等人 ${ }^{[52]}$ 证明, DNA 甲基化具有组织差异性 的特点. 随后一系列的研究揭示了DNA甲基化在小 肠细胞分化过程中的作用. 首先, 通过基因组水平的 DNA 甲基化图谱揭示了 DNA CpG 岛的甲基化是在小 肠干细胞的分化过程中的一种动态表观遗传学标记, 虽然只有 50 个甲基化区域存在显著差异, 也说明DNA 甲基化动态变化在小肠干细胞分化中具有一定的作 用; 而转录因子Tcf4(TCF4 transcription factor 4)的结合 与低甲基化现象相关联, 则证明了 Tcf4的结合是形成 甲基化区域差异性的一个重要原因 ${ }^{[33]}$. 其次, 发现了 增强子上DNA低甲基化在小肠干细胞自我增殖过程 中是必要的 ${ }^{[54]}$; 条件性敲除小鼠肠道甲基转移酶基因 (methyltransferase 1, Dnmt1), 发现小肠隐窝增生 ${ }^{[55]}$, 在 沿着小肠干细胞分化方向存在DNA 甲基化改变, 其中 主要是形成超甲基化现象, 并且在基因组中超甲基化 的富集与基因调控紧密联系 ${ }^{[56]}$.

\section{4 小肠上皮细胞更新及损伤修复的营养调控}

肠上皮细胞功能的变化必然引起营养素代谢的 变化, 而产生的代谢物也在一定程度上调控肠上皮细 胞的增殖分化和肠道发育. 现有的研究表明, EGF、 转化生长因子(transforming growth factor, $\mathrm{TGF}$ )、氨基 酸及其衍生物和核苷酸等营养因子以及代谢产物分 别通过不同的作用机制调控哺乳动物肠道上皮细胞 增殖分化和损伤后的重新修复.

\subsection{EGF}

EGF 是由 53 个氨基酸残基组成的单链小分子多 肽, 在胃肠道等组织分布较丰富. 外源性EGF能促进 小鼠肠黏膜辐射损伤后小肠隐窝细胞的快速增生, 证
明了足够量的外源性 EGF 能促进小肠干细胞的增殖 和分化, 以此来修复受损的肠上皮细胞 ${ }^{[57]}$. 使用表达 EGF的乳酸菌发酵上清液饲喂早期断奶仔猪, 发现能 够促进新生仔猪的肠道发育, 其中杯状细胞的数量, 线毛的高度和抗炎症因子IL-13的表达都显著增加 ${ }^{[58]}$. 另外, $\mathrm{EGF}$ 在小肠内能增加肠谷氨酰胺酶活性, 为小 肠提供能量及酰胺氮以促进细胞增殖 ${ }^{[59]}$. 而肠道隐 窝-线毛轴上的内源EGF信号也能促进小肠干细胞和 短暂扩增细胞 (TA 细胞) 的增殖 ${ }^{[60]}$; 其作用机制是特 异性结合细胞膜上的糖蛋白受体(EGFR), 而后受体 磷酸化, 激活酪氨酸激酶, 开启一系列下游信号通路 (如PI3K/Akt和Ras/Raf/MEK/ERK等通路)作用于细胞 核内转录因子, 通过调节下游基因的转录来调控细胞 的增殖和分化 ${ }^{[61]}$. 在隐窝干细胞中, EGF的下游通路 $\mathrm{Ras} / \mathrm{Raf} / \mathrm{MEK} / \mathrm{ERK}$ 是处于激活状态的 ${ }^{[62]}$.

\section{$4.2 \quad$ TGF- $\beta$}

TGF- $\beta$ 是一种产生于整个胃肠道的多肽, 在肠道 黏膜上皮细胞的更新、损伤修复的调控以及维持肠道 细胞完整性方面发挥了重要作用. 外源性TGF- $\beta 1$ 可通 过活化丝裂原活化蛋白激酶(mitogen-activated protein kinases, MAPK)、Smad信号通路增加细胞紧密连接蛋 白的表达量, 从而加强肠黏膜免疫屏障功能, 缓解肠黏 膜炎症及通透性增加 ${ }^{[63]}$. 内源性TGF- $\beta$ 在肠道上皮细 胞的增殖分化、促进细胞修复方面也具有重要调节 作用; 作为 TGF- $\beta$ 超家族的重要成员—BMP, 参与了 调控细胞的增殖、分化和调亡等诸多过程, 在胚胎发 育和成体干细胞维持中发挥着重要的作用 ${ }^{[64]}$. 由BMP 信号控制小肠上皮细胞发育分化过程中的稳态的机 制已经得到阐明; 早期胚胎肠发育过程中, 内胚层诱 导信号BMP-4在内壁中胚层表达, 从而调节中胚层细 胞增殖 ${ }^{[65]}$; 随后, BMPs在线毛生长之前介导了线毛和 隐窝周边间充质浓缩 ${ }^{[66,67]}$; 在成熟的肠道中, BMPs主 要控制肠上皮细胞增生 ${ }^{[68]}$. 进一步的研究证明, 激活 的BMP信号是通过细胞内的PTEN/PI-3K/AKT和Smad 等途径直接抑制 $\beta$-catenin的代谢和活性 ${ }^{[69]}$, 从而拮抗 Wnt信号通路, 维持上皮细胞增殖与分化的动态平衡. 而隐窝基底层和周边细胞会分泌Noggin和Chordin等 BMP的拮抗分子来抑制隐窝附近的BMP信号,使干细 胞周围的BMP保持较低的浓度, 促进肠隐窝干细胞的 自我更新和不断增殖 ${ }^{[70,71]}$. 


\section{3 氨基酸极其衍生物}

在肠道干细胞的增殖和分化过程中, 氨基酸通 过激活多条信号通路来共同完成调节. $L$-谷氨酰胺 ( $L$-glutamine, Gln) 作为非必需氨基酸, 是肠道的主要 功能物质. Gln缺乏时可诱导鼠肠上皮细胞发生调亡, 从而促进肠道干细胞的分化来形成新的上皮细胞 ${ }^{[72]}$. 补充Gln能通过上调紧密连接蛋白的表达来促进细胞 生长和维持细胞膜的完整性, 提高氧化应激反应和黏 膜屏障功能 ${ }^{[73]}$. 在 $\mathrm{Gln}$ 补给肠切除的动物模型实验中 发现Gln能特异增加肠黏膜生长因子(EGF和IGF-II)的 表达; 而EGF, IGF-II能通过激活一系列信号通路促进 肠道干细胞的增殖和分化, 重建受损的肠道上皮 ${ }^{[74]}$. Marc Rhoads和 $\mathrm{Wu}^{[75]}$ 发现, Gln能激活MAPK; 在 Gln补 给对大鼠肠道损伤恢复的实验发现, Gln 能显著促进 P-ERK的表达 ${ }^{[76]}$, 上述研究说明, Gln在激活ERK信号 通路的同时, 也通过增加生长因子的表达来促进肠道 干细胞的增殖和分化, 从而修复受损的肠上皮. 通过饲 粮添加 Gln和丙氨酰谷氨酰胺二肽(Ala-Gln)对LPS 诱 导的仔猪应激实验结果显示, 在肠腔和血浆中 Gln浓度 增加, 肠道细胞中半胱氨酸蛋白酶-3(caspase-3)活性, 核转录因子 $\kappa \mathrm{B}$ (nuclear facter of $\kappa \mathrm{B}, N F-\kappa B$ ) 和 Toll样受 体4(Toll-like receptor 4,TLR4)的表达显著降低, 从而预 防和降低肠道氧化损伤和炎症, 增强细胞生长性能 [77].

$L$-精氨酸 $(L$-arginine, $A r g)$ 作为一种碱性必需氨基 酸, 在促进动物肠道生长和结构改善, 加速受损黏膜 修复, 维护肠道屏障功能方面有重要作用. 研究证明, Arg与感应器(CASTOR1)结合后激活雷帕霉素靶蛋白 (mTOR)/P70(S6)激酶通路, 增强黏膜细胞迁移和损伤 恢复 ${ }^{[75,78]}$. 本研究组通过在饲粮中添加Arg 可提高断奶 仔猪肠道热休克蛋白 70(heat shock protein 70, HSP70) mRNA和蛋白的表达, 增加肠道黏膜杯状细胞的数量, 提高营养物质利用率 ${ }^{[79]}$; 并发现补充Arg 可增加断奶 仔猪肠道线毛高度和黏膜血管内皮生长因子水平, 降 低皮质醇、氨(NH3)和尿素浓度, 提高血浆胰岛素和 $\operatorname{Arg}$ 浓度, 促进肠道发育 ${ }^{[80]}$.

$\mathrm{N}$-乙酰半胱氨酸 (N-acetyl- $L$-cysteine, NAC) 是一 种强有效的抗氧化剂, 在断奶仔猪饲粮中添加NAC后 发现, 肠道黏膜抗调亡基因 $B c l-2$, 细胞周期调控基因 $E 2 F 5, S m a d 4$ 表达显著上调, 而调亡相关基因死亡受体 (factor associated suicide, Fas)和创伤愈合指示基因整合
素 $\alpha v \beta 6($ alpha $\mathrm{v}$ beta 6 integrin)表达显著下调, 表明NAC 在一定程度上可以缓减仔猪断奶应激, 提高仔猪抗氧化 能力, 保护肠细胞结构的完整性 ${ }^{[81]}$. 饲粮中添加NAC也 可缓解LPS刺激导致的仔猪肠黏膜中的免疫因子(IL-2 和IL-6)和细胞紧密连接蛋白(occludin和claudin-1)表达 升高, 并降低小肠黏膜细胞中 caspase- 3 的表达, 有效缓 解肠道黏膜损伤 ${ }^{[82]}$. 另外, 赖氨酸 ${ }^{[83]}$ 、蛋氨酸 ${ }^{[84]} 、 \alpha-$ 酮戊二酸 ${ }^{[85}$ 等在对维持肠黏膜形态和功能的完整性 以及肠上皮细胞的增殖有一定的促进作用.

\section{4 外源性核苷酸}

外源性核苷酸对小肠细胞分化也有重要影响, 通 过核苷混合物(胞苷、尿苷、鸟苷和肌苷)对大鼠小肠 隐窝上皮细胞(IEC-6)进行培养; 结果发现尿苷、鸟苷 和肌苷在培养基中消失, 而胞苷浓度增加; 且分化的 IEC-6细胞中核苷酸的浓度显著升高. 这些变化同时伴 随着碱性磷酸酶活性的增加, 线毛形态的延长和 $R N D 3$ 基因表达下调. 表明外源核苷酸选择性的被IEC-6细 胞吸收, 增加了细胞内的核苷酸量、GTP和能荷, 有利 于分化过程中细胞的形态和功能变化 ${ }^{[86]}$. 在体内实验 中, 核苷酸显著提高早期断奶大鼠肠黏膜Invertase活 性; 结果表明, 核苷酸的补充对提高小肠上皮细胞体 内和体外的增殖和成熟有促进作用, 因此, 外源核苷 酸可能在肠道细胞更新中起着重要的作用 ${ }^{[87]}$

\section{5 其他}

脂肪酸, 糖类除了作为肠黏膜细胞主要能量来源 外, 还能减少炎症发生, 促进肠细胞代谢、增殖和分 化. 饲粮中添加丁酸钠可增加仔猪肠黏膜厚度和肠黏 膜上线毛高度和隐窝深度的比值, 并促进结肠内杯状 细胞的分化, 增强肠道消化吸收功能 ${ }^{[88]}$. 本研究组通 过在饲粮中添加壳多糖可增强肠黏膜紧密连接蛋白 (occludin和ZO-1)的表达, 提高肠黏膜紧密连接性, 阻 缓肠黏膜通透性升高, 并对大肠杆菌攻毒的早期断奶 仔猪小肠黏膜屏障功能的损伤有保护作用 ${ }^{[89]}$; 补充黄 芪多糖(astragalus polysacharin, APS)能够促进断奶仔 猪的肠道细胞更新, 改善肠道消化功能、调节氨基酸 代谢, 从而达到促生长的目的 ${ }^{[00]}$. 另外, 在饲粮中添加 适宜的营养性微量元素锌 $(\mathrm{Zn})$ 能通过促进肠黏膜分泌 型免疫球蛋白 $\mathrm{A}(\mathrm{SIgA})$ 和IL-2的表达来维持肠黏膜免 
疫屏障的稳定性, 进而促进肠道细胞的分化 ${ }^{[91]}$.

\section{5 总结和展望}

众所周知, 小肠上皮的更新需要隐窝基底部干细 胞不断增殖和分化的维持. 到目前为止, 随着对小肠 隐窝干细胞增殖分化机制的研究, 逐步发现了包括多 种营养因子和Wnt信号通路等一系列信号在小肠细胞 的增殖和更新中发挥着重要的调控作用, 而这些信号 的异常与肠道疾病发生有着密切的关系. 虽然人们对
小肠细胞自我更新的调控有一定的了解,但是对于调 控小肠干细胞增殖和分化信号通路的具体机制, 肠道 营养物质的吸收量对肠上皮细胞分化与细胞结构变 化的影响, 以及干细胞分化与肠上皮细胞功能之间的 关系等许多问题还没有解决. 总之, 对小肠上皮细胞 自我更新调控机制进行深入研究, 不仅可以使人们更 加了解其内在的分子调控机制, 更能在营养学和药理 学方面, 为干预和治疗肠道损伤及相关疾病提供新的 线索和依据。

\section{参考文献}

1 Wu G. Intestinal mucosal amino acid catabolism. J Nutr, 1998, 128: 1249-1252

2 van der Flier L G, Clevers H. Stem cells, self-renewal, and differentiation in the intestinal epithelium. Annu Rev Physiol, 2009, 71: 241-260

3 Xiong X, Yang H, Hu X, et al. Differential proteome analysis along jejunal crypt-villus axis in piglets. Front Biosci, 2016, 21: 343-363

4 Qi Z, Chen Y G. Regulation of intestinal stem cell fate specification. Sci China Life Sci, 2015, 58: 570-578

5 Barker N, van de Wetering M, Clevers H. The intestinal stem cell. Genes Dev, 2008, 22: 1856-1864

6 Zhu M H, Sung T S, Kurahashi M, et al. $\mathrm{Na}^{+}-\mathrm{K}^{+}-\mathrm{Cl}^{-}$cotransporter (NKCC) maintains the chloride gradient to sustain pacemaker activity in interstitial cells of Cajal. Am J Physiol Gastrointest Liver Physiol, 2016, 311: G1037-G1046

7 Fan M Z, Stoll B, Jiang R, et al. Enterocyte digestive enzyme activity along the crypt-villus and longitudinal axes in the neonatal pig small intestine. J Animal Sci, 2001, 79: 371-381

8 Cheng H, Leblond C P. Origin, differentiation and renewal of the four main epithelial cell types in the mouse small intestine V. Unitarian theory of the origin of the four epithelial cell types. Am J Anat, 1974, 141: 537-561

9 Engelstoft M S, Egerod K L, Lund M L, et al. Enteroendocrine cell types revisited. Curr Opin Pharmacol, 2013, 13: $912-92$

10 Neutra M R. Current concepts in mucosal immunity. V Role of M cells in transepithelial transport of antigens and pathogens to the mucosal immune system. Am J Physiol, 1998, 274: G785-G791

11 Gerbe F, Legraverend C, Jay P. The intestinal epithelium tuft cells: specification and function. Cell Mol Life Sci, 2012, 69: 2907-2917

12 Canale-Zambrano J C, Poffenberger M C, Cory S M, et al. Intestinal phenotype of variable-weight cystic fibrosis knockout mice. Am J Physiol Gastrointestinal Liver Physiol, 2007, 293: G222-G229

13 Günther C, Neumann H, Neurath M F, et al. Apoptosis, necrosis and necroptosis: cell death regulation in the intestinal epithelium. Gut, 2013, 62: $1062-1071$

14 Turan A, Mahmood A. The profile of antioxidant systems and lipid peroxidation across the crypt-villus axis in rat intestine. Dig Dis Sci, 2007, 52: $1840-1844$ small intestine. Am J Physiol Gastrointest Liver Physiol, 2015, 309: G229-G237

19 Rexroad C E 3rd, Rodriguez M F, Coulibaly I, et al. Comparative mapping of expressed sequence tags containing microsatellites in rainbow trout (Oncorhynchus mykiss). BMC Genomics, 2005, 6: 54-56

20 Jiang L, David E S, Espina N, et al. GLUT-5 expression in neonatal rats: crypt-villus location and age-dependent regulation. Am J Physiol Gastrointest Liver Physiol, 2001, 281: G666-G674

21 Yang C, Albin D M, Wang Z, et al. Apical $\mathrm{Na}^{+}-D$-glucose cotransporter 1 (SGLT1) activity and protein abundance are expressed along the jejunal crypt-villus axis in the neonatal pig. Am J Physiol Gastrointestinal Liver Physiol, 2011, 300: G60-G70 
jejunum. Biochim Biophys Acta, 2009, 1790: 1624-1635

23 Levy E, Ménard D, Delvin E, et al. Localization, function and regulation of the two intestinal fatty acid-binding protein types. Histochem Cell Biol, 2009, 132: 351-367

24 Montagne L, Boudry G, Favier C, et al. Main intestinal markers associated with the changes in gut architecture and function in piglets after weaning. Br J Nutr, 2007, 97: 45-57

25 Zhu L H, Xu J X, Zhu S W, et al. Gene expression profiling analysis reveals weaning-induced cell cycle arrest and apoptosis in the small intestine of pigs. J Animal Sci, 2014, 92: 996-1006

26 Hou Y. Gene expression profiles in the intestine of lipopolysaccharide-challenged piglets. Front Biosci, 2016, 21: 487-501

27 Zhu L H, Zhao K L, Chen X L, et al. Impact of weaning and an antioxidant blend on intestinal barrier function and antioxidant status in pigs. J Animal Sci, 2012, 90: 2581-2589

28 Pinto D, Gregorieff A, Begthel H, et al. Canonical Wnt signals are essential for homeostasis of the intestinal epithelium. Genes Dev, 2003, 17: 1709-1713

29 VanDussen K L, Carulli A J, Keeley T M, et al. Notch signaling modulates proliferation and differentiation of intestinal crypt base columnar stem cells. Development, 2012, 139: 488-497

30 Yin X, Mead B E, Safaee H, et al. Engineering stem cell organoids. Cell Stem Cell, 2016, 18: 25-38

31 Yin X, Farin H F, van Es J H, et al. Niche-independent high-purity cultures of grr $^{5+}$ intestinal stem cells and their progeny. Nat Methods, 2013, 11: $106-112$

32 Mariadason J M, Nicholas C, L'Italien K E, et al. Gene expression profiling of intestinal epithelial cell maturation along the crypt-villus axis. Gastroenterology, 2005, 128: 1081-1088

33 Mochizuki K, Yagi E, Sakaguchi N, et al. The critical period for thyroid hormone responsiveness through thyroid hormone receptor isoform $\alpha$ in the postnatal small intestine. Biochim Biophys Acta, 2007, 1770: 609-616

34 West A R, Oates P S. Decreased sucrase and lactase activity in iron deficiency is accompanied by reduced gene expression and upregulation of the transcriptional repressor PDX-1. Am J Physiol Gastrointestinal Liver Physiol, 2005, 289: G1108-G1114

35 Zuo Z, Urban G, Scammell J G, et al. Ser/Thr protein phosphatase type 5 (PP5) is a negative regulator of glucocorticoid receptor-mediated growth arrest. Biochemistry, 1999, 38: 8849-8857

36 Chawengsaksophak K, James R, Hammond V E, et al. Homeosis and intestinal tumours in Cdx2 mutant mice. Nature, 1997, 386: 84-87

37 Shimakura J, Terada T, Shimada Y, et al. The transcription factor Cdx2 regulates the intestine-specific expression of human peptide transporter 1 through functional interaction with Sp1. Biochem Pharmacol, 2006, 71: 1581-1588

38 Kakizaki F, Aoki K, Miyoshi H, et al. CDX transcription factors positively regulate expression of solute carrier family 5 , member 8 in the colonic epithelium. Gastroenterology, 2010, 138: 627-635

39 Yamamoto H, Miyamoto K I, Li B, et al. The caudal-related homeodomain protein Cdx-2 regulates vitamin D receptor gene expression in the small intestine. J Bone Miner Res, 1999, 14: 240-247

40 Naito Y, Oue N, Hinoi T, et al. Reg IV is a direct target of intestinal transcriptional factor CDX2 in gastric cancer. PLoS ONE, 2012, 7: e47545

41 Coskun M, Boyd M, Olsen J, et al. Control of intestinal promoter activity of the cellular migratory regulator gene ELMO3 by CDX2 and SP1. J Cell Biochem, 2010, 109: 1118-1128

42 Li X G, Xu G F, Zhai Z Y, et al. CDX2 increases SLC7A7 expression and proliferation of pig intestinal epithelial cells. Oncotarget, 2014, 7: 30597-30609

43 Fang R, Olds L C, Sibley E. Spatio-temporal patterns of intestine-specific transcription factor expression during postnatal mouse gut development. Gene Expr Patterns, 2006, 6: 426-432

44 Beuling E, Bosse T, aan de Kerk D J, et al. GATA4 mediates gene repression in the mature mouse small intestine through interactions with friend of GATA (FOG) cofactors. Dev Biol, 2008, 322: 179-189

45 Gao X, Sedgwick T, Shi Y B, et al. Distinct functions are implicated for the GATA-4, -5 , and -6 transcription factors in the regulation of intestine epithelial cell differentiation. Mol Cell Biol, 1998, 18: 2901-2911

46 Beuling E, Baffour-Awuah N Y A, Stapleton K A, et al. GATA factors regulate proliferation, differentiation, and gene expression in small intestine of mature mice. Gastroenterology, 2011, 140: 1219-1229.e2

47 Walker E M, Thompson C A, Battle M A. GATA4 and GATA6 regulate intestinal epithelial cytodifferentiation during development. Dev Biol, 2014, 392: 283-294

48 Benoit Y D, Paré F, Francoeur C, et al. Cooperation between HNF-1 $\alpha$, Cdx2, and GATA-4 in initiating an enterocytic differentiation program in a normal human intestinal epithelial progenitor cell line. Am J Physiol Gastrointestinal Liver Physiol, 2010, 298: G504-G517 
, Peinato gene expression in the villus. Genome Biol, 2013, 14: R50 Cells Dev, 2015, 24: 2733-2739

55 Sheaffer K L, Kim R, Aoki R, et al. DNA methylation is required for the control of stem cell differentiation in the small intestine. Genes Dev, 2014, 28: 652-664

56 Forn M, Díez-Villanueva A, Merlos-Suárez A, et al. Overlapping DNA methylation dynamics in mouse intestinal cell differentiation and early stages of malignant progression. PLoS ONE, 2015, 10: e0123263

57 Potten C, Booth D, Haley J. Pretreatment with transforming growth factor beta-3 protects small intestinal stem cells against radiation damage in vivo. Br J Cancer, 1997, 75: 1454-1459

58 Bedford A, Chen T, Huynh E, et al. Epidermal growth factor containing culture supernatant enhances intestine development of early-weaned pigs in vivo: potential mechanisms involved. J Biotech, 2015, 196-197: 9-19

59 Wang J Y, Zhang L H, Song W L. Epidermal growth factor regulates intestinal glutamine uptake during total parenteral nutrition. Clin Nutr, 1996, 15: 21-23

60 Marchbank T, Goodlad R A, Lee C Y, et al. Luminal epidermal growth factor is trophic to the small intestine of parenterally fed rats. Clin Sci, 1995, 89: $117-120$

61 Waseem T, Duxbury M, Ashley S W, et al. Ghrelin promotes intestinal epithelial cell proliferation through PI3K/Akt pathway and EGFR transactivation both converging to ERK 1/2 phosphorylation. Peptides, 2014, 52: 113-121

62 Wong V W Y, Stange D E, Page M E, et al. Lrig1 controls intestinal stem-cell homeostasis by negative regulation of ErbB signalling. Nat Cell Biol, 2012, 14: 401-408

63 Howe K L, Reardon C, Wang A, et al. Transforming growth factor- $\beta$ regulation of epithelial tight junction proteins enhances barrier function and blocks enterohemorrhagic Escherichia coli O157:H7-induced increased permeability. Am J Pathol, 2005, 167: 1587-1597

64 Zhang J, Li L. BMP signaling and stem cell regulation. Dev Biol, 2005, 284: 1-11

65 Roberts D J, Smith D M, Goff D J, et al. Epithelial-mesenchymal signaling during the regionalization of the chick gut. Development, 1998, 125: 2791-2801

\section{Kosinski C, Li V S W, Chan A S Y, et al. Gene expression patterns of h
cell niche factors. Proc Natl Acad Sci USA, 2007, 104: 15418-15423}

71 Li X, Madison B B, Zacharias W, et al. Deconvoluting the intestine: molecular evidence for a major role of the mesenchyme in the modulation of signaling cross talk. Physiol Genomics, 2007, 29: 290-301

72 Papaconstantinou H T, Hwang K O, Rajaraman S, et al. Glutamine deprivation induces apoptosis in intestinal epithelial cells. Surgery, 1998, 124: 152-160; discussion 159-160

73 Jiao N, Wu Z, Ji Y, et al. L-glutamate enhances barrier and antioxidative functions in intestinal porcine epithelial cells. J Nutr, 2015, 145: 2258-2264

74 Wirén M, Adrian T E, Arnelo U, et al. Early gastrointestinal regulatory peptide response to intestinal resection in the rat is stimulated by enteral glutamine supplementation. Dig Surg, 1999, 16: 197-203

Swaid F, Sukhotnik I, Matter I, et al. Dietary glutamine supplementation prevents mucosal injury and modulates intestinal epithelial restitution following acetic acid induced intestinal injury in rats. Nutr Metab (Lond), 2013, 10: 53

77 Haynes T E, Li P, Li X, et al. $L$-Glutamine or $L$-alanyl- $L$-glutamine prevents oxidant- or endotoxin-induced death of neonatal enterocytes. Amino Acids, 2009, 37: 131-142 
78 Chantranupong L, Scaria S M, Saxton R A, et al. The CASTOR proteins are arginine sensors for the mTORC1 pathway. Cell, 2016, 165: 153-164

79 Wu X, Ruan Z, Gao Y, et al. Dietary supplementation with 1-arginine or N-carbamylglutamate enhances intestinal growth and heat shock protein-70 expression in weanling pigs fed a corn- and soybean meal-based diet. Amino Acids, 2010, 39: 831-839

80 Yao K, Guan S, Li T, et al. Dietary l-arginine supplementation enhances intestinal development and expression of vascular endothelial growth factor in weanling piglets. Br J Nutr, 2011, 105: 703-709

81 Zhu L, Cai X, Guo Q, et al. Effect of N-acetyl cysteine on enterocyte apoptosis and intracellular signalling pathways' response to oxidative stress in weaned piglets. Br J Nutr, 2013, 110: 1938-1947

82 Hou Y, Wang L, Zhang W, et al. Protective effects of N-acetylcysteine on intestinal functions of piglets challenged with lipopolysaccharide. Amino Acids, 2012, 43: 1233-1242

83 He L, Yang H, Hou Y, et al. Effects of dietary $L$-lysine intake on the intestinal mucosa and expression of $C A T$ genes in weaned piglets. Amino Acids, 2013, 45: 383-391

84 Shen Y B, Weaver A C, Kim S W. Effect of feed grade $L$-methionine on growth performance and gut health in nursery pigs compared with conventional $D L$-methionine. J Animal Sci, 2014, 92: 5530-5539

85 Yao K, Yin Y, Li X, et al. Alpha-ketoglutarate inhibits glutamine degradation and enhances protein synthesis in intestinal porcine epithelial cells. Amino Acids, 2012, 42: 2491-2500

86 María Vieites J, Torre R, Ramírez M D C, et al. Exogenous nucleosides accelerate differentiation of rat intestinal epithelial cells. Br J Nutr, 2008, 99: $732-738$

87 Sato N, Morimoto H, Baba R, et al. Functional expression of double-stranded RNA-dependent protein kinase in rat intestinal epithelial cells. J Cell Biochem, 2010, 110: 104-111

88 Manzanilla E G, Nofrarías M, Anguita M, et al. Effects of butyrate, avilamycin, and a plant extract combination on the intestinal equilibrium of early-weaned pigs. J Animal Sci, 2006, 84: 2743-2751

89 Xiao D, Tang Z, Yin Y, et al. Effects of dietary administering chitosan on growth performance, jejunal morphology, jejunal mucosal sIgA, occluding, claudin-1 and TLR4 expression in weaned piglets challenged by enterotoxigenic Escherichia coli. Int Immunopharmacol, 2013, 17: $670-676$

90 Yin F G, Liu Y L, Yin Y L, et al. Dietary supplementation with Astragalus polysaccharide enhances ileal digestibilities and serum concentrations of amino acids in early weaned piglets. Amino Acids, 2009, 37: 263-270

91 Starke I C, Pieper R, Neumann K, et al. The impact of high dietary zinc oxide on the development of the intestinal microbiota in weaned piglets. FEMS Microbiol Ecol, 2014, 87: 416-427 


\title{
Current advances in renewal mechanisms of intestinal epithelial cells along the crypt-villus axis
}

\author{
ZOU LiJun ${ }^{1,2,3}$, XIONG Xia ${ }^{2}$, WANG XiaoCheng ${ }^{2}$, YANG HuanSheng ${ }^{1}$, LI \\ JianZhong ${ }^{1}$, HUANG PengFei ${ }^{1} \&$ YIN YuLong ${ }^{1,2}$ \\ 1 Laboratory of Animal Nutrition and Human Health, College of Life Sciences, Hunan Normal University, Changsha 410081, China; \\ 2 Key Laboratory for Agro-Ecological Processes in Subtropical Regions, Institute of \\ Subtropical Agriculture, the Chinese Academy of Sciences, Changsha 410125, China, \\ 3 Laboratory of Basic Biology, Hunan First Normal College Changsha, 410205, China
}

\begin{abstract}
The small intestine is not only an organ for food digestion and nutrient absorption but also an integral part of the immune and endocrine systems. Differentiation of enterocytes plays an important role in the damage repair mechanism after immune response elicitation by the intestine, for restoring the intestinal barrier function and homeostasis. Recently, research related to self-renewal, differentiation, and regulation of the intestinal epithelial crypt stem cells has been developing rapidly. In this review, we attempt to sum up recent studies related to the expression pattern of genes and proteins along the crypt-villus axis, and the effect of signaling pathways, transcription factors, and epigenetic modifications on the differentiation of crypt stem cells. We also examine the mechanisms by which nutritional factors influence the differentiation and damage repair processes of the intestinal epithelial cells. This review could provide valuable theoretical framework in nutritional and pharmacological aspects and guidance for intervention and treatment of intestinal damage and related diseases.
\end{abstract}

small intestine, crypt-villus axis, cell renewal, damage repair, nutrition regulation

doi: $10.1360 / \mathrm{N} 052016-00240$ 